\title{
A QUADRATIC EIGENVALUE PROBLEM
}

\author{
W. M. GREENLEE ${ }^{1}$
}

Abstract. Let $P, Q$ be compact selfadjoint operators in a Hilbert space. It is proven that the characteristic and associated vectors of the quadratic eigenvalue problem, $x=\lambda P x+(1 / \lambda) Q x$, form a Riesz basis for the cartesian product of the closure of the range of $P$ and the closure of the range of $Q$.

1. Investigations in the theory of hydrodynamic stability (cf. [3, Chapter X]; [8]) lead to the search for expansion theorems associated with the following characteristic value problem. Let $P, Q$ be compact selfadjoint operators in a Hilbert space $H$ and for $x \in H$ let

$$
A(\lambda) x=\lambda P x+(1 / \lambda) Q x .
$$

$\sigma$, the spectrum of $A(\lambda)$, is defined as the set of all complex numbers $\lambda$ for which $I-A(\lambda)$ does not have a bounded inverse defined on all of $H . \rho$, the resolvent of $A(\lambda)$, is the complement of $\sigma$ in the complex plane. A number $\lambda_{0} \neq 0$ is a characteristic value of $A(\lambda)$ if the equation

$$
x=A\left(\lambda_{0}\right) x=\lambda_{0} P x+\left(1 / \lambda_{0}\right) Q x
$$

has nonzero solutions. Any such $x$ is called a characteristic vector of $A(\lambda)$ corresponding to $\lambda=\lambda_{0}$.

Since any nonzero number on the imaginary axis is in $\rho$, it follows that $\sigma$ is at most countable (cf. [5, p. 21]). All nonzero points in $\sigma$ are isolated characteristic values whose only possible limit points are 0 and $\infty$. Thus, via the transformation $\lambda \rightarrow a \lambda, a>0$, it may be assumed without loss of generality that $\pm 1 \in \rho$. Following [4] the characteristic values are poles of the function $(I-A(\lambda))^{-1}$, which is analytic on $\rho$.

Presented to the Society, January 17, 1972 under the title Bases associated with a quadratic eigenvalue problem; received by the editors September 25, 1972.

AMS (MOS) subject classifications (1970). Primary 47B50, 35P10, 76E99; Secondary 46D05.

Key words and phrases. Quadratic eigenvalue problem, completeness, indefinite metric, Rayleigh-Taylor problem.

${ }^{1}$ This research was supported in part by NSF Grants GP-19816 and GP-28484. A presentation of this material was given at the Seminar on Nonlinear Eigenvalue Problems, Santa Fe, New Mexico, June 22, 1971. 
Now let $\lambda_{0}$ be a characteristic value of $A(\lambda)$ and let $m+1$ be the order of the pole of $(I-A(\lambda))^{-1}$ at $\lambda_{0}$. The vectors $x_{1}, x_{2}, \cdots, x_{i}$ are called associated vectors of the characteristic vector $x_{0}$, corresponding to $\lambda_{0}$, if

$$
\begin{aligned}
A\left(\lambda_{0}\right) x_{k}+\frac{1}{1 !} \frac{d A}{d \lambda}\left(\lambda_{0}\right) x_{k-1}+\cdots+\frac{1}{k !} \frac{d^{k} A}{d \lambda^{k}}\left(\lambda_{0}\right) x_{0} & =x_{k} ; \\
k & =1, \cdots, i \leqq m .
\end{aligned}
$$

Again, following [4] all characteristic values are of finite multiplicity and therefore any root subspace (i.e. the span of all characteristic and associated vectors corresponding to a characteristic value) corresponding to any characteristic value is finite dimensional. Then as in [2], all nonreal characteristic values of $A(\lambda)$, and all real characteristic values corresponding to characteristic vectors having associated vectors, are in the ring

$$
(1 / 2\|P\|) \leqq|\lambda| \leqq 2\|Q\|
$$

2. We wish now to state and sketch the proof of an expansion theorem for the characteristic value problem (1). The proof is obtained by modifying the linearization procedure of G. I. Laptev which is carried out in [2]. The modification symmetrizes the linearized problem. This obviates the necessity of the assumption that $P, Q$ be of finite order in [2], and also gains an expansion theorem via an orthonormality property of all but a finite number of the characteristic vectors of the linearized problem. A more detailed exposition and further results will appear elsewhere.

THEOREM. The system of characteristic and associated vectors $x_{n, k}$ corresponding to characteristic values $\lambda_{n}$ of $A(\lambda)$ is "doubly complete" in $H$ in the following sense: for any pair of vectors $x, y$ in $H$ there are expansions

$$
\sum_{n, k} c_{n, k} x_{n, k} \rightarrow x
$$

in the seminorm $\left\|P_{1} x\right\|$, where $P_{1}$ is the orthogonal projection on the closure of the range of $P$, and

$$
\sum_{n, k} c_{n, k} \sum_{j=0}^{k} \frac{(-1)^{j}}{\lambda_{n}^{j+1}} x_{n, k-j} \rightarrow y
$$

in the seminorm $\left\|Q_{1} x\right\|$, where $Q_{1}$ is the orthogonal projection on the closure of the range of $Q$. These expansions are unconditional.

3. To linearize problem (1), observe that (1) is equivalent to each of the equations,

$$
x=\left(\lambda-\frac{1}{\lambda}\right) P x+\frac{1}{\lambda}(Q+P) x ; \quad \frac{1}{\lambda} x=(P+Q) x-\frac{1}{\lambda}\left(\lambda-\frac{1}{\lambda}\right) Q x .
$$


Via the change of variables $\mu=\lambda-(1 / \lambda), y=(1 / \lambda) x$, these equations can be considered as the single equation in $H \times H$,

$$
Y=\mathscr{R} Y+\mu \mathscr{H} Y,
$$

where

$\mathscr{R}=\left(\begin{array}{cc}0 & P+Q \\ P+Q & 0\end{array}\right), \quad \mathscr{H}=\left(\begin{array}{cc}P & 0 \\ 0 & -Q\end{array}\right), \quad$ and $\quad Y=\left(\begin{array}{l}x \\ y\end{array}\right) \in H \times H$.

Let $\mathscr{I}$ denote the identity operator in $H \times H$. Both $\mathscr{R}$ and $\mathscr{H}$ are compact selfadjoint operators in $H \times H$ and, by the previous remark that without loss of generality $\pm 1 \in \rho, \mathscr{I}-\mathscr{R}$ may be assumed invertible. So (2) is equivalent to the characteristic value problem

$$
\mu(\mathscr{I}-\mathscr{R})^{-1} \mathscr{H} Y=Y .
$$

Now the characteristic and associated vectors of $A(\lambda)$, corresponding to a characteristic value $\lambda_{0}$, transform into characteristic and associated vectors of $(\mathscr{I}-\mathscr{R})^{-1} \mathscr{H}$, corresponding to the characteristic value $\mu_{0}=$ $\lambda_{0}-\left(1 / \lambda_{0}\right)$, which are of special form

$$
\left(\begin{array}{c}
x_{k} \\
\sum_{j=0}^{k} \frac{(-1)^{j}}{\lambda_{0}^{j+1}} x_{k-j}
\end{array}\right), \quad k=0,1, \cdots, i,
$$

where $x_{0}$ is a characteristic vector and $x_{1}, \cdots, x_{i}$ associated vectors of $x_{0}$.

To obtain a converse, let $\mathscr{T}(\mu)=\mathscr{R}+\mu \mathscr{H}$ and for $\lambda \neq \pm i$ introduce the operator

Then:

$$
\mathscr{D}(\lambda)=\frac{1}{\lambda^{2}+1}\left(\begin{array}{ll}
\lambda^{2} & \lambda \\
\lambda & 1
\end{array}\right)
$$

and

$$
\mathscr{D}^{2}(\lambda)=\mathscr{D}(\lambda), \quad \mathscr{I}-\mathscr{D}(\lambda)=\mathscr{D}(-1 / \lambda),
$$

$$
\mathscr{D}(\lambda) \mathscr{T}(\lambda-(1 / \lambda))=\mathscr{T}(\lambda-(1 / \lambda)) \mathscr{D}(\lambda) .
$$

It follows that $H \times H$ can be decomposed into a direct sum of two subspaces invariant with respect to $\mathscr{T}(\lambda-(1 / \lambda))$ by use of the two complementary projections $\mathscr{D}(\lambda)$ and $\mathscr{D}(-1 / \lambda)$. It now follows as in [2] that every root subspace of the operator $(\mathscr{I}-\mathscr{R})^{-1} \mathscr{H}$ has a basis consisting of vectors of special form.

Let $\mathscr{P}_{0}$ be the orthogonal projection in $H \times H$ onto the null space of $\mathscr{H}$, and let $\mathscr{P}=\mathscr{I}-\mathscr{P}_{0}$. Letting $Y_{0}=\mathscr{P}_{0} Y$ and $Y_{1}=\mathscr{P} Y$, (2) becomes

$$
Y_{0}+Y_{1}=\mathscr{R} Y_{0}+\mathscr{R} Y_{1}+\mu \mathscr{H} Y_{1} \text {. }
$$


Applying $\mathscr{P}_{0}$ and $\mathscr{P}$ to this equation yields

$$
Y_{0}=\mathscr{P}_{0} \mathscr{R} Y_{1}, \quad Y_{1}=\mathscr{F} Y_{1}+\mu \mathscr{H} Y_{1},
$$

where $\mathscr{F}$ is a compact selfadjoint operator acting in $\mathscr{P}(H \times H)$. Then via the transformation $\lambda \rightarrow a \lambda$ with $a>0$, it may be assumed without loss of generality that $\mathscr{I}-\mathscr{F}$ is invertible on $\mathscr{P}(H \times H)$.

Now completeness (in $\mathscr{P}(H \times H))$ of the characteristic and associated vectors of $(\mathscr{I}-\mathscr{F})^{-1} \mathscr{H}$ is equivalent to completeness of the characteristic and associated vectors of the similar operator $\mathscr{A}=\mathscr{H}(\mathscr{I}-\mathscr{F})^{-1}$. So let (cf. [5, pp. 263-265])

$$
\mathscr{J}=(\mathscr{I}-\mathscr{F})^{-1}, \quad\{X, Z\}=(\mathscr{J} X, Z) .
$$

With respect to the Hermitian symmetric bilinear form $\{X, Z\}, \mathscr{A}$ is selfadjoint. If the eigenvalues of $\mathscr{J}$ are greater than zero, completeness of the characteristic vectors of $\mathscr{A}$ and a $\mathscr{J}$-orthonormal basis result. Otherwise, suppose $\mathscr{J}$ has eigenvalues less than zero of total multiplicity $\kappa$. Then completeness follows from a theorem of [6]. Actually (cf. [7]) there exists a direct sum decomposition

$$
\mathscr{P}(H \times H)=\mathscr{L}+\mathscr{N}
$$

where $\mathscr{L}$ and $\mathscr{N}$ are closed subspaces each invariant with respect to $\mathscr{A}$. Furthermore the dimension of $\mathscr{L}$ is not more than $2 \kappa$, the quadratic form $\{X, X\}$ is positive definite on $\mathscr{N}$ and $\mathscr{A}$ has in $\mathscr{N}$ a $\mathscr{J}$-orthonormal system of characteristic vectors corresponding to real characteristic values. The structure of the root subspaces of $\mathscr{A}$ in $\mathscr{L}$ is analyzed in [9].

4. If $P$ and $Q$ are both nonnegative (or nonpositive), except possibly on a finite dimensional subspace, then $A(\lambda)$ has a sequence of characteristic values converging to $\infty$ (resp. 0 ) if and only if $P$ (resp. $Q$ ) has a sequence of characteristic values (resp. nonzero eigenvalues) tending to $\infty$ (resp. 0). Then if $A(\lambda)$ has a sequence of characteristic values converging to $\infty$ or 0 , these characteristic values are asymptotic to characteristic values or to negative reciprocals of characteristic values of (2).

5. As an example consider an equation derived in [3, Chapter 10], in the study of the Rayleigh-Taylor problem:

$$
\begin{gathered}
D\left\{\left[\rho-(\mu / n)\left(D^{2}-k^{2}\right)\right] D w-(1 / n)(D \mu)\left(D^{2}+k^{2}\right) w\right\} \\
=k^{2}\left\{-\left(g / n^{2}\right)(D \rho) w+\left[\rho-(\mu / n)\left(D^{2}-k^{2}\right)\right] w-(2 / n)(D \mu) D w\right\} \\
w(0)=D w(0)=w(d)=D w(d)=0
\end{gathered}
$$

where $g$ and $k^{2}$ are positive constants, $\rho$ and $\mu$ are given positive functions of $z$, and $D=d / d z$. The characteristic value parameter is $n$ and, if

$$
\left(\inf D^{2} \mu\right)>-2(\inf \mu)\left\{\left[\min \left(d^{2}, d^{4} / 12\right)\right]^{-1 / 2}+d^{-1}\right\},
$$


then the fourth order differential operator occurring as the coefficient of $1 / n$ is of definite sign and this problem can be converted to the form (1). Presumably stability results for the Rayleigh-Taylor problem should be obtainable if $D \rho \leqq 0$. Stability if $D \rho<0$ and instability if $D \rho>0$ on a set of positive measure is claimed in [1], but no proofs are given. A full expansion for the associated initial value problem has been carried out in [10] when $D \rho>0$, an unstable case.

\section{REFERENCES}

1. Dž. Ė. Allahverdiev, Multiply complete systems and nonselfadjoint operators depending on a parameter $\lambda$, Dokl. Akad. Nauk SSSR 166 (1966), 11-14=Soviet Math. Dokl. 7 (1966), 4-8. MR 33 \#7657.

2. N. K. Askerov, S. G. Krein and G. I. Laptev, Oscillations of a viscous liquid and the associated operational equations, Funkcional. Anal. i Priložen 2 (1968), no. 2, 21-31= Functional Anal. Appl. 2 (1968), 115-124. MR 38 \#559.

3. S. Chandrasekhar, Hydrodynamic and hydromagnetic stability, Internat. Ser. of Monographs on Physics, Clarendon Press, Oxford, 1961. MR 23 \#B1270.

4. A. Friedman and M. Shinbrot, Nonlinear eigenvalue problems, Acta Math. 121 (1968), 77-125. MR 40 \#3337.

5. I. C. Gohberg and M. G. Krein, Introduction to the theory of linear nonselfadjoint operators, "Nauka," Moscow, 1965; English transl., Transl. Math. Monographs, vol. 18, Amer. Math. Soc., Providence, R.I., 1969. MR 36 \#3137; MR 39 \#7447.

6. I. S. Iohvidov, On the spectra of Hermitian and unitary operators in a space with indefinite metric, Dokl. Akad. Nauk SSSR 71 (1950), 225-228. (Russian) MR 12, 33.

7. I. S. Iohvidov and M. G. Kreìn, Spectral theory of operators in spaces with an indefinite metric. I, Trudy Moskov. Mat. Obšč. 5 (1956), 367-432; English transl., Amer. Math. Soc. Transl. (2) 13 (1960), 105-175. MR 18, 320; MR 22 \#3983.

8. S. G. Kreĭn and G. I. Laptev, Motion of a viscous liquid in an open vessel, Funkcional Anal. i Priložen. 2 (1968), no. 1, 40-50=Functional Anal. Appl. 2 (1968), 38-47. MR 40 \#1714.

9. A. I. Mal'cev, Foundations of linear algebra, OGIZ, Moscow, 1948, 1956; English transl., Freeman, San Francisco, Calif., 1963. MR 11, 412; MR 29 \#3477.

10. R. E. L. Turner, A problem in Rayleigh-Taylor instability, Rend. Sem. Mat. Univ. Padova 42 (1969), 305-323. MR 41 \#5800.

Department of Mathematics, Northwestern University, Evanston, Illinois 60201

Current address: Department of Mathematics, University of Arizona, Tucson, Arizona 85721 